\title{
Morphology and Biochemistry of Microbial Antagonists and Their Effect in the Growth of Garbanzo and Control of Fusariosis
}

\section{Luz del Carmen Oliva Ortiz¹, Teresa de Jesús Velázquez Alcaraz¹, Rogelio Sosa Pérez², Leopoldo Partida Ruvalcaba3*, Tomás Díaz Valdés' ${ }^{1}$, Julio Arciniega Ramos', Jacobo Enrique Cruz Ortega ${ }^{1}$}

${ }^{1}$ Facultad de Agronomía, Universidad Autónoma de Sinaloa, Culiacán de Rosales, México

${ }^{2}$ Centron de Ciencias de Sinaloa, Culiacán de Rosales, México

${ }^{3}$ Universidad Tecnológica de Culiacán, Culiacán de Rosales, México

Email: *parpolo@yahoo.com.mx

How to cite this paper: Oliva Ortiz, L.C., Velázquez Alcaraz, T.J., Pérez, R.S., Ruvalcaba, L.P., Valdés, T.D., Ramos, J.A. and Ortega, J.E.C. (2016) Morphology and Biochemistry of Microbial Antagonists and Their Effect in the Growth of Garbanzo and Control of Fusariosis. Open Access Library Journal, 3: e3226.

http://dx.doi.org/10.4236/oalib.1103226

Received: November 11, 2016

Accepted: December 3, 2016

Published: December 6, 2016

Copyright $\odot 2016$ by authors and Open Access Library Inc.

This work is licensed under the Creative Commons Attribution International

License (CC BY 4.0).

http://creativecommons.org/licenses/by/4.0/

(c) (i) Open Access

\section{Abstract}

The chickpea (Cicer arietinum L.) is an important crop in the world. In this study, six strains of indigenous rhizosphere microorganisms (T442, T3241, 7A1, 751, HRG050 y HRG-060) were characterized morphologically and biochemically through catalogs and API tests, and evaluated as growth promoters and biocontrol agents of $F$. oxysporum f. sp. ciceris ( $F o c$ ), causal agent of Fusarium wilt of chickpea under laboratory and field conditions. Preliminary studies were performed in vitro, evaluating antagonism by PICR of Foc, in Petri dishes with PDA culture medium; the stimulation of germination was evaluated by seed inoculation with the same strains. In the field, chickpea seeds were inoculated with said antagonistic strains at a concentration of $1 \times 10^{8}$ ufc or conidia $\mathrm{mL}^{-1}$, establishing eight treatments: six based on the inoculation of seed with each antagonist strain, a chemical treatment (Benomyl) and a witness, in block design randomized complete, evaluating the protective effect and stimulatory in chickpea plants. The strains were characterized as Bacillus subtilis, Agrobacterium radiobacter, Pseudomonas sp., Trichoderma sp., and Bacillus megaterium, the latter with low percentage of identification. HRG-060 (Trichoderma sp.) and T442 (Bacillus subtilis) proved to be the best biocontrol agents in laboratory and field, as well as higher growth promoters, as significantly reduced growth of the pathogen and the incidence of the disease in the field, and increased the germination, greenness, biomass and yield of chickpea. The use of strains HRG-060 y T442 to control Fusarium wilt of chickpea may be possible in the conditions that have the central area of Sinaloa. 


\section{Subject Areas}

Plant Science

\section{Keywords}

Trichoderma, Bacillus, Fusarium oxysporum ciceris Race 5, Native Strains

\section{Introduction}

El garbanzo (Cicer arietinum L.), es un cultivo de gran importancia en la alimentación mundial, debido a principalmente a su alto contenido de proteína [1]; siendo la segunda leguminosa más importante del mundo después del frijol [2]. Su principal productor y consumidor es la India, con una cosecha de 9,356,250 t en el ciclo 20132014, ocupando México el octavo lugar de producción, al aportar 190,803 t en ese mismo ciclo [3]; llevándose a cabo su producción principalmente en la zona Noroeste del país, que comprende a Sinaloa y Sonora [4], siendo Sinaloa el principal productor y exportador de garbanzo [5]. La fusariosis vascular es la enfermedad más importante que afecta al garbanzo en todo el mundo [2], la cual reduce su rendimiento [6], y ha sido reportada en la mayoría de las zonas donde se cultiva garbanzo [2]. Uno de los principales agentes causales de esta enfermedad es Fusarium oxysporum f. sp. ciceris (Foc) [7]; en el centro de Sinaloa se ha reportado a Foc raza 5 como el principal patógeno [8]. En los últimos años, las pérdidas debidas a la fusariosis vascular han variado del $10 \%$ - 70\%, significando reducciones de exportación, incremento de costos de producción y disminución de la rentabilidad [4]. El uso de fungicidas en el tratamiento a la semilla es una forma de reducir esta enfermedad [9]; sin embargo, ningún tratamiento químico ha proporcionado un nivel de control satisfactorio [10]. Además, estos compuestos producen efectos negativos en el medio ambiente, pues contaminan el suelo, agua y aire, generando residuos tóxicos, además de inducir generaciones de fitopatógenos resistentes, alterando el equilibrio ecológico [11] [12]. Debido a los resultados adversos que producen los pesticidas, la tendencia en la agricultura es la utilización de insumos de bajo impacto ambiental y sin problemas de toxicidad [13]; situando así al control biológico como una alternativa dentro del manejo de enfermedades [14].

Numerosos estudios se han realizado con diversos microorganismos rizosféricos para el biocontrol de F. oxysporum [15] [16]; identificándose algunos antagonistas con efecto de supresión en Foc y que mejoran el crecimiento del garbanzo como bacterias del género Bacillus y Pseudomonas, así como hongos del género Trichoderma [10] [11]. Cuyos principales mecanismos de control biológico son la competencia por nutrientes y espacio, antibiosis, parasitismo, producción de compuestos inhibidores e inducción de resistencia sistémica en la planta [15] [17] [18]. La antibiosis de Trichoderma es por producción de sustancias como tricodermina, tricodermol, tricotoxina, dermadina [19] [20]; mientras que Bacillus y Pseudomonas producen metabolitos antifúngicos como: 
iturinas, bacitracina, bacillin, subtenolin, ácido cianhídrico, entre otros [16] [21]. El parasitismo de estos tres agentes biocontrol, es inducido por la producción de enzimas de degradación de la pared celular, como: celulasas, quitinasas, glucanasas y proteasas [22] [23]. Asimismo, estos organismos son considerados como promotores del crecimiento vegetal y contribuyen a la producción de sustancias como fitohormonas, metabolitos secundarios, fitoalexinas y sideróforos [17] [22] [23]; pueden favorecer el crecimiento de la planta por medio de la fijación de nitrógeno, solubilización y absorción de fósforo y otros nutrientes, incrementan el desarrollo de la raíz, estimulan la germinación, emergencia de plántulas e incrementan la tolerancia al estrés [17] [22] [23] [24].

Asimismo, es tema de interés, el estudio de antagonistas autóctonos, pues el principal problema en la efectividad de biocontrol de patógenos, es la introducción de organismos no nativos en los ecosistemas, ya que no se encuentran adaptados a las condiciones ambientales donde se van a aplicar [25], señalándose que el empleo de cepas comerciales pudiera generar competencia o inhibición contra los organismos, o simplemente no prosperar su propagación dentro de la microbiota del suelo [26].

Los objetivos de esta investigación fueron caracterizar morfológica y bioquímicamente a seis microorganismos autóctonos previamente aislados de raíces de plantas de garbanzo, cultivado en suelos con problemas de fusariosis vascular ubicados en la parte central de Sinaloa; determinar en condiciones de laboratorio y campo su potencial antagonista contra Foc raza 5, principal agente causal de la enfermedad; así como estimar su efecto como promotores de crecimiento y rendimiento en garbanzo.

\section{Materiales y Métodos}

\subsection{Localización Geográfica}

Esta investigación se realizó en el campo experimental del Instituto Nacional de Investigación y Fomento Agrícola y Pecuario (INIFAP, Culiacán), con coordenadas geográficas de $24^{\circ} 37^{\prime} 49^{\prime \prime}$ latitud Norte y $107^{\circ} 26^{\prime} 17^{\prime \prime}$ longitud Oeste, así como en el laboratorio del Centro de Ciencias de Sinaloa. Se seleccionó como parcela experimental un predio infestado fuertemente desde hace varios años por fusariosis vascular del garbanzo, en la cual se realizó un muestreo de suelo en forma al azar y un análisis de fertilidad del suelo, cuyas características se presentan en la Table 1, observándose que es un suelo de textura arcillosa, de $\mathrm{pH}$ neutro, sin problemas de sales y bajo contenido de materia orgánica, con capacidad de intercambio catiónico media, altas concentraciones de calcio y magnesio, contenido medio de potasio y niveles bajos de nitratos, fósforo y azufre. Las concentraciones de los nutrientes solubles e intercambiables son bajas a excepción del calcio y magnesio, cuya concentración es alta y adecuada respectivamente.

\subsection{Activación y Caracterización de las Cepas Antagonistas Microbianas}

Se realizaron ensayos in vitro y en campo con seis microorganismos antagonistas 
Table 1. Análisis de fertilidad del suelo de la parcela experimental.

\begin{tabular}{|c|c|c|c|}
\hline \multicolumn{2}{|c|}{ Análisis } & \multicolumn{2}{|c|}{ Resultados } \\
\hline \multicolumn{2}{|c|}{ Textura } & \multicolumn{2}{|c|}{$15 \%$ arena, $16 \%$ limo, $69 \%$ arcilla } \\
\hline \multicolumn{2}{|c|}{$\mathrm{pH}$} & \multicolumn{2}{|c|}{7.1} \\
\hline \multicolumn{2}{|c|}{$\mathrm{CE}$} & \multicolumn{2}{|c|}{$1.76 \mathrm{dS} \cdot \mathrm{m}^{-1}$} \\
\hline \multicolumn{2}{|c|}{ Materia orgánica } & \multicolumn{2}{|c|}{$1.12 \%$} \\
\hline \multicolumn{2}{|c|}{$\mathrm{CIC}$} & \multicolumn{2}{|c|}{22.43 meq $100 \mathrm{~g}$ suelo } \\
\hline \multicolumn{2}{|c|}{ Contenido nutrimental total (ppm) } & \multicolumn{2}{|c|}{ Nutrientes solubles $\left(\mathrm{meq} \cdot \mathrm{L}^{-1}\right)$} \\
\hline $\mathrm{N}-\mathrm{NO}_{3}$ total & 13.7 & $\mathrm{NO}_{3}^{-}$ & 0.89 \\
\hline P total & 4.8 & $\mathrm{P}-\mathrm{PO}_{4}^{2-}$ & 0.02 \\
\hline$S$ total & 5.0 & $\mathrm{~S}-\mathrm{SO}_{4}^{2-}$ & 0.63 \\
\hline K intercambiable & 332.0 & $\mathrm{~K}^{+}$ & 0.41 \\
\hline Ca intercambiable & 3346.0 & $\mathrm{Ca}^{2+}$ & 8.86 \\
\hline Mg intercambiable & 425.0 & $\mathrm{Mg}^{2+}$ & 2.45 \\
\hline Na intercambiable & 320.0 & $\mathrm{Na}^{+}$ & 5.85 \\
\hline
\end{tabular}

autóctonos del centro de Sinaloa, México; cuatro bacterias (T442, T3141, 751, 7A1) y dos hongos (HRG-050 y HRG-060) del cepario del Centro de Ciencias de Sinaloa, aislados previamente a partir de suelo rizosférico de plantas de garbanzo con síntomas de fusariosis. Para evaluar el antagonismo in vitro de estas cepas, se empleó una cepa de Fusarium oxysporum f. sp. ciceris raza 5, principal agente de la fusariosis del garbanzo en esta zona, perteneciente a la colección del INIFAP, identificada molecularmente e ingresada al GeneBank como KJ000584-raza 5 [8]. Las cepas fueron activadas mediante la inoculación de cajas Petri con medio de cultivo a base de agar nutritivo (AN) y agar FLO (AF) para las bacterias por el método de asada gruesa; y las cepas fúngicas en medio papa dextrosa agar (PDA), por el método de porción y fueron incubados a $28^{\circ} \mathrm{C}$ durante $48 \mathrm{~h}$ en el caso de las bacterias y por cinco días en los hongos, en una incubadora de mesa Thermo Scientific, Modelo BK6160.

En principio, las cepas fueron sometidas a una distinción morfológica colonial [27], para ello se sembraron por medio de asada, en cajas Petri con medio de cultivo de AN y AF para las bacterias y en PDA las cepas fúngicas, siendo incubadas a $28^{\circ} \mathrm{C}$ por 48 y 72 horas, respectivamente. En las cepas bacterianas se determinaron las características microscópicas, considerando tinción Gram y presencia de esporas mediante la técnica de Schaeffer-Fulton [28]; para la identificación mediante su conducta bioquímica se empleó el sistema de diagnóstico API BioMérieux 50CHB V4.0 complementado con 20NE V7.0; para ello se hicieron inóculos con material biológico fresco, el cual fue suspendido en solución salina y con éllo se llenaron las galerías de los API, y fueron incubadas a $33^{\circ} \mathrm{C}$ por 24 y 48 horas, observándose posteriormente los colores de las reacciones producidas; una vez obtenidos los perfiles, éstos fueron identificados 
utilizando la base de datos de BioMérieux, empleada en estos ensayos. Para la identificación de las cepas fúngicas se tomaron en cuenta características morfológicas de sus estructuras macroscópicas (color de micelio, forma de micelio y crecimiento) y microscópicas (hifas, conidios, clamidospora), considerando las claves taxonómicas de Ainsworth [29].

\subsection{Confrontaciones in Vitro}

Se realizaron bioensayos in vitro para determinar el efecto antagónico de las cepas por medio de la técnica de cultivo dual [30], en cajas Petri $(90 \times 15 \mathrm{~mm})$ con medio de cultivo PDA (15 mL); un disco de $1.0 \mathrm{~cm}$ de diámetro del micelio del fitopatógeno, con previo crecimiento ( 6 días) en PDA debido a las diferencias en sus tasas de crecimiento, fue sembrado a un $\mathrm{cm}$ de la orilla de la placa y fueron incubados a $28{ }^{\circ} \mathrm{C}$; tres días después se sembró por el método de asada gruesa al centro de la caja, cada uno de los antagonistas bacterianos [31]; los antagonistas fúngicos fueron sembrados por el método de porción, al otro extremo de la caja, mediante discos del mismo diámetro que el patógeno, tomados de la parte con esporulación de la colonia del antagonista [31]. Además se contó con un testigo absoluto en caja Petri con medio PDA, se colocó a 1.0 $\mathrm{cm}$ del borde un disco de $5 \mathrm{~mm}$ de diámetro del micelio del patógeno. Se realizaron cuatro réplicas por cada cepa. Las placas se incubaron a $28^{\circ} \mathrm{C}$ por 15 días. El crecimiento radial de las colonias de antagonistas y fitopatógeno se midió cada $24 \mathrm{~h}$, realizando las observaciones hasta los diez de post-incubación. La capacidad antagónica por antibiosis de las cepas ensayadas fue evaluada a los siete días de post-inoculación, mediante el Porcentaje de Inhibición del Crecimiento Radial (PICR), empleando la fórmula propuesta por [32], PICR $=[(R 1-R 2) / 100]$; donde, PICR fue el porcentaje de inhibición en el crecimiento del micelio del fitopatógeno; $R 1$ fue el crecimiento de la colonia del patógeno-testigo; y $R 2$ el crecimiento radial de la colonia del patógeno en confrontación en cultivo dual.

\subsection{Preparación del Inóculo Bacteriano y Fúngico}

Matraces de $500 \mathrm{~mL}$ con medio nutritivo a base de caldo nutritivo y caldo soya triptocaseína para bacterias y PDA para los hongos fueron inoculados con cada cepa. Mediante un asa se tomó una muestra de cada antagonista, previamente activado y purificado, colocándose en los matraces con los medios correspondientes; una vez inoculados se pasaron a una incubadora a $28^{\circ} \mathrm{C}$ con agitación $150 \mathrm{RPM}$, durante una semana, para obtener la biomasa que fue cosechada por medio de una centrifuga Sigma 3-18P (20 minutos a $3800 \mathrm{RPM}$ ), a temperatura ambiente. Las bacterias se cuantificaron por la técnica de diluciones seriadas en tubos con solución salina estéril al $0.85 \%$, y por conteo de colonias en placa, empleando la escala de McFarland, con turbiedad igual al estándar 0.5 [33], para obtener una suspensión con una concentración de $1 \times 10^{8}$ UFC $\mathrm{mL}^{-1}$. La concentración de esporas fúngicas se estimó utilizando una cámara de Neubauer, para una concentración de $1 \times 10^{8}$ conidios $\mathrm{mL}^{-1}$ [34]. Con este inóculo se impregnaron semillas de garbanzo para efectuar la prueba de germinación de semillas y 
para la siembra en el ensayo de campo.

\subsection{Ensayo de Germinación de Semillas en Laboratorio}

Con cada cepa antagonista (T442, T3141, 751, 7A1, HRG-050 y HRG-060) fueron inoculadas 40 semillas de garbanzo, colocándose diez semillas por caja Petri, además se contó con un testigo absoluto, donde a las semillas sólo se les aplicó agua, por tanto fueron siete tratamientos conformados con cuatro repeticiones; la unidad experimental consistió en una caja Petri. Una vez inoculadas las semillas, se pusieron en cuarto oscuro a temperatura ambiente para observar la germinación.

\subsection{Ensayo en Campo}

El trabajo se llevó a cabo durante el ciclo 2013-2014, la siembra se efectuó en Diciembre de 2013, empleándose semillas de garbanzo variedad Blanco Sinaloa 92. Cada tratamiento se aplicó en seis surcos de ocho $\mathrm{m}$ de largo. La densidad de siembra fue de doce plantas por metro lineal a una distancia de $10 \mathrm{~cm}$ entre semillas, con una separación de $0.80 \mathrm{~m}$ entre surcos y una profundidad de siembra de $15 \mathrm{~cm}$. Para la fertilización se aplicaron los siguientes productos: nitrato de potasio, nitrato de calcio, sulfato de magnesio, fosfato monopotásico en dosis de 10, 10, 4 y $2 \mathrm{~kg} \cdot \mathrm{ha}^{-1}$, respectivamente. Se aplicó un riego de auxilio a los 20 días después de la siembra.

\subsection{Tratamientos y Diseño Experimental en Campo}

Se establecieron en base a la inoculación de semillas de garbanzo con las cepas autóctonas T442, T3141, 7A1, 751, HRG-050 y HRG-060, un fungicida químico y el testigo. Se estableció un diseño experimental de bloques completos al azar, con ocho tratamientos y seis bloques por tratamiento. En cada bloque se escogieron diez plantas para evaluar el efecto protector y estimulatorio del crecimiento de los tratamientos. Para determinar el efecto protector de los antagonistas se empleó escala subjetiva, con valores de 1 a 5 para evaluar las variables vigor de planta (donde 1 representaba $0 \%$ de vigor y 5 significaba $100 \%$ de vigor, considerando desarrollo y flacidez de la planta); marchitez de follaje y cáncer oscuro en raíz (donde 1 era el $0 \%$ de clorosis en el follaje y $0 \%$ de cáncer en raíz, y 5 era el $100 \%$ de follaje totalmente marchito y $100 \%$ de raíces con cáncer oscuro). Para determinar el efecto de la estimulación del crecimiento y rendimiento, las variables consideradas fueron: altura de planta $(\mathrm{cm})$, empleando cinta metálica desde la base del tallo hasta la parte más alta de la planta; diámetro de tallo $(\mathrm{mm})$ medido con vernier digital, a $1.0 \mathrm{~cm}$ de la base del tallo; verdor de plantas en unidades del medidor Spad-502, marca Spectrum Technologies, Inc.; peso fresco y peso seco de planta (g); rendimiento $(\mathrm{g})$ planta $^{-1}$.

\subsection{Análisis Estadístico}

Todos los datos se sometieron a un Análisis de Varianza, después de comprobarse su normalidad y homogeneidad, y se aplicó la prueba de comparación múltiple de medias de Tukey $(P \leq 0.05)$ en SAS Versión 1.3. 


\section{Resultados y Discusión}

\subsection{Caracterización de Las Cepas Antagonistas}

En la Table 2 se indica la descripción morfológica de las cepas, según el Manual de Bergey's, Palleroni [35]. En base a las características presentadas, se pudo otorgar una previa distinción, para posteriormente aplicar las pruebas bioquímicas, Larrea et al. [36]; las colonias de T442 y T3141 se ubicaron previamente dentro del género de Bacillus por su forma irregular, color crema y apariencia de los bordes, Sosa et al. [37]; 7A1 y 751 se clasificaron como Pseudomonas, Palleroni [35]; y las cepas fúngicas se identificaron dentro del género Trichoderma, Kirk and Ainsworth [29].

\subsection{Características Microscópicas de Cepas Bacterianas}

Con la prueba de tinción de Gram se logró conocer que las cepas T442 y T3141 mostraron tinción violeta, indicando ser células Gram positivas, además presentaron forma bacilar y presencia de endosporas; 7AI y 751 presentaron tinción Gram negativa, con forma de bacilos ligeramente curvados y sin presencia de esporas, lo que a su vez permitió corroborar que corresponden a Bacillus y Pseudomonas, respectivamente, según el Manual de Bergey's, Palleroni [35]; resultados que son similares a los encontrados por Larrea et al. [36] para determinar el género Bacillus, así como por Álvarez et al. [38], quienes mediante esta prueba identificaron a los microorganismos empleados como Pseudomonas.

\subsection{Identificación de Las Cepas Bacterianas Mediante su Respuesta Bioquímica}

La caracterización bioquímica de las bacterias se presenta en la Table 3, en donde se

Table 2. Descripción de la morfología colonial de los antagonistas microbianos.

\begin{tabular}{|c|c|c|c|c|c|c|}
\hline Característica & T442 & T3141 & $7 \mathrm{~A} 1$ & 751 & HRG-050 & HRG-060 \\
\hline Diámetro colonial & $2 \mathrm{~mm}$ & $3 \mathrm{~mm}$ & $2 \mathrm{~mm}$ & $3 \mathrm{~mm}$ & $12 \mathrm{~mm}$ & $18 \mathrm{~mm}$ \\
\hline Forma & Irregular & Irregular & Circular & Irregular & Circular & Circular \\
\hline Borde & $\begin{array}{c}\text { Ligeramente } \\
\text { lobulado }\end{array}$ & Liso & Dentado & Liso & Filamentoso & Filamentoso \\
\hline Superficie & Lisa & Lisa & Lisa & Lisa & Lascinada & Lascinada \\
\hline Consistencia & Butirosa & Mucosa & Húmeda & Butirosa & Seca & Seca \\
\hline Color & Crema & Blanquecina & Crema & Blanca & Verde & Verde \\
\hline Color UV & - & - & $\begin{array}{l}\text { Amarillo } \\
\text { verdoso }\end{array}$ & Cremoso & - & - \\
\hline Olor & Ácido láctico & Ácido láctico & Ácido láctico & Heces & $\begin{array}{l}\text { Tierra } \\
\text { Húmeda }\end{array}$ & $\begin{array}{c}\text { Tierra } \\
\text { húmeda }\end{array}$ \\
\hline Brillo & Opaco & Brillante & Brillante & Opaco & Opaco & Opaco \\
\hline Paso de luz & Mate & Mate & Mate & Mate & Mate & Mate \\
\hline Elevación & Plana & $\begin{array}{l}\text { Ligeramente } \\
\text { convexa }\end{array}$ & $\begin{array}{c}\text { Ligeramente } \\
\text { convexa }\end{array}$ & Plana & Papilada & Papilada \\
\hline
\end{tabular}


Table 3. Pruebas bioquímicas de las cepas bacterianas antagonistas.

\begin{tabular}{|c|c|c|c|c|c|c|c|c|}
\hline \multirow{2}{*}{$\begin{array}{c}\text { API } 50 \text { CHB } \\
\text { Reacción }\end{array}$} & \multicolumn{2}{|c|}{ Cepa } & \multirow{2}{*}{$\begin{array}{c}\text { API } 50 \text { CHB } \\
\text { Reacción }\end{array}$} & \multicolumn{2}{|c|}{ Cepa } & \multirow{2}{*}{$\begin{array}{c}\text { API } 20 \text { NE } \\
\text { Reacción }\end{array}$} & \multicolumn{2}{|c|}{ Cepa } \\
\hline & T3141 & T442 & & T3141 & T442 & & 7A1 & 751 \\
\hline 0 & - & - & ESC & + & + & NO3 & + & + \\
\hline GLY & + & + & SAL & + & + & TRP & - & - \\
\hline ERY & - & - & CEL & + & + & GLU & - & - \\
\hline DARA & - & - & MAL & + & + & $\mathrm{ADH}$ & - & - \\
\hline LARA & + & + & LAC & + & + & URE & + & + \\
\hline RIB & + & + & MEL & + & + & ESC & + & + \\
\hline DXYL & + & + & SAC & + & + & GEL & - & + \\
\hline LXYL & - & - & TRE & + & + & PNG & + & + \\
\hline $\mathrm{ADO}$ & - & - & INU & - & - & GLU & + & + \\
\hline MDX & - & - & MLZ & - & - & ARA & + & + \\
\hline GAL & - & + & RAF & + & + & MNE & + & + \\
\hline GLU & + & + & AMD & + & + & MAN & + & + \\
\hline FRU & + & + & GLYG & + & + & NAG & + & + \\
\hline MNE & + & + & XLT & - & - & MAL & + & + \\
\hline SBE & - & - & GEN & - & + & GNT & + & + \\
\hline RHA & - & - & TUR & - & - & CAP & + & - \\
\hline DUL & - & - & LYX & - & - & $\mathrm{ADI}$ & + & - \\
\hline INO & + & + & TAG & - & - & MLT & + & + \\
\hline MAN & + & + & DFUC & - & - & CIT & + & - \\
\hline SOR & + & + & LFUC & - & - & PAC & + & - \\
\hline $\mathrm{MDM}$ & - & - & DARL & - & - & OX & + & + \\
\hline MDG & + & + & LAPL & - & - & & & \\
\hline NAG & - & + & GNT & - & - & & & \\
\hline AMY & + & + & $2 \mathrm{KG}$ & - & - & & & \\
\hline ARB & + & + & $5 \mathrm{KG}$ & - & - & & & \\
\hline
\end{tabular}

puede observar que las cepas de T3141 y T442 sólo difirieron en las pruebas con Galactoxa (GAL), N-Acetil Glucosamina (NAG) y Gentobiosa (GEN), siendo negativas para T3141 y positivas para T442. Mientras que en las cepas 751 y 7AI las diferencias se presentaron con las pruebas de Hidrólisis de la Gelatina (GEL), asimilación de Ácido Cáprico (CAP), Ácido Adípico (ADI), Citrato (CIT) y Ácido Fenil Acético (PAC); en la primera la reacción fue negativa para 7AI y positiva para 751, con las otras cuatro pruebas 7AI presentó reacciones positivas y las de 751 fueron negativas.

Los análisis de perfil bioquímico aportados por la base de datos APILAB del test API $50 \mathrm{CHB}$ y API $20 \mathrm{NE}$, ayudan a caracterizar de manera fácil y rápida la fisiología de estas bacterias, Gacitúa et al. [39], considerándose muy útiles para la identificación 
hasta el nivel de especie por su elevada precisión. Además de la observación de morfología y esporulación, la respuesta a la tinción de Gram permite ubicarlos dentro de su género, Sosa et al. [37] y Álvarez et al. [38]. Mediante el empleo de los sistemas API, los microorganismos pudieron ser ubicados con un alto porcentaje de identificación (Table 4), aunque para la cepa T3141 dicho porcentaje fue bajo.

\subsection{Ubicación Taxonómica de Las Cepas Fúngicas}

El análisis morfológico efectuado con el microscopio biológico mostró que estas cepas pertenecen al género Trichoderma sp., de acuerdo al Catálogo de Ainsworth [29], encontrándose micelio septado, presencia de conidióforo hialino muy ramificado, fiálides individuales y en grupos, conidios ovoides, pequeños racimos terminales de color verde fuerte a verde olivo y clamidosporas intercalares típicas del género Trichoderma.

\subsection{Confrontaciones in Vitro}

El efecto antagonista in vitro de los agentes biocontroladores del crecimiento micelial de $F$. oxysporum ciceris se muestran en la Table 5. La capacidad antagónica de las cepas ocurrió con diferencias significativas $(P<0.05)$, observándose la formación de tres grupos, el primero integrado por HRG-060 y HRG-050 con el mayor efecto inhibitorio ( $82.67 \%$ y $78.33 \%$ ), respectivamente; el segundo integrado por T442 y 751, las cuales inhibieron al fitopatógeno en los respectivos $58.7 \%$ y $57.3 \%$; y el tercero que formaron T3141 y 7AI, que inhibieron al patógeno en 34.7\% y 33.3\%, respectivamente.

Table 4. Identificación taxonómica de las cepas bacterianas.

\begin{tabular}{ccc}
\hline Cepa & Taxón significativo & $\% \mathrm{ID}$ \\
\hline $\mathrm{T} 442$ & Bacillus subtilis & 99.9 \\
$\mathrm{~T} 3141$ & Bacillus megaterium & 50.8 \\
751 & Agrobacterium radiobacter & 96.6 \\
$7 \mathrm{~A} 1$ & Pseudomonas sp. & 90.0 \\
\hline
\end{tabular}

\%ID = porcentaje de identificación.

Table 5. Crecimiento radial in vitro de Fusarium oxysporum ciceris raza 5, al ser inhibidos por los antagonistas.

\begin{tabular}{ccc}
\hline Cepas & Crecimiento del patógeno $(\mathrm{cm})$ & PICR $(\%)$ \\
\hline T3141 & 4.9 & $34.66 \mathrm{c}^{*}$ \\
T442 & 3.1 & $58.67 \mathrm{~b}$ \\
751 & 3.2 & $57.33 \mathrm{~b}$ \\
$7 \mathrm{~A} 1$ & 5.0 & $33.33 \mathrm{c}$ \\
HRG-060 & 1.3 & $82.67 \mathrm{a}$ \\
HRG-050 & 1.6 & $78.33 \mathrm{a}$ \\
\hline
\end{tabular}

${ }^{\star}$ Letras diferentes en la columna indican diferencias estadísticamente significativas, según Tukey $(P<0.05)$. 
Tanto en los antagonistas fúngicos como bacterianos, los valores de inhibición del crecimiento del patógeno fueron variados, siendo unos altamente inhibitorios mientras que en otros fue limitada, sugiriendo que hay una variación entre el tipo de metabolitos antifúngicos producidos por las cepas, concordando con los resultados obtenidos por Eshetu et al. [16] y López et al. [40].

Los resultados logrados con las cepas HRG-060 y HRG-050 (Trichoderma sp.) son muy semejantes a los obtenidos por Aponte et al. [41] con cepas ensayadas para el biocontrol in vitro de diferentes patógenos del suelo, incluido Fusarium; también coinciden con los de Ríos et al. [42], quienes reportan inhibición de crecimiento de $F$. oxysporum en más del 50\% con cepas nativas de Trichoderma (Figure 1). De las cepas bacterianas, la que ocasionó mayor PICR (58.7\%) fue T442, identificada bioquímicamente como Bacillus subtilis, valor que de acuerdo con Gajbhiye et al. [43] es competitivo, ya que en su investigación aislaron e identificaron cepas de B. subtilis de la rizósfera de algodón y fueron probadas como agentes biocontrol de F. oxysporum, inhibiendo su micelio en más de un $50 \%$.

Mediante diversas investigaciones se ha demostrado que con aplicaciones de Trichoderma y Bacillus subtilis se obtienen excelentes resultados de biocontrol contra diferentes enfermedades que afectan a diversos cultivos. Así, Moradi et al. [11] evaluaron cepas nativas de Trichoderma y B. subtilis, las cuales suprimieron eficazmente la marchitez vascular del garbanzo causada por F. oxysporum ciceris. Asimismo, Kumar et al. [44] trabajaron con aislados de Trichoderma y Bacillus en confrontación con $F$. oxysporum lycoperscisi y reportaron que el porcentaje de inhibición del patógeno por Trichoderma osciló en $44.4 \%$ - 84.8\%, y entre $45.1 \%$ - $63.4 \%$ al emplear aislados de

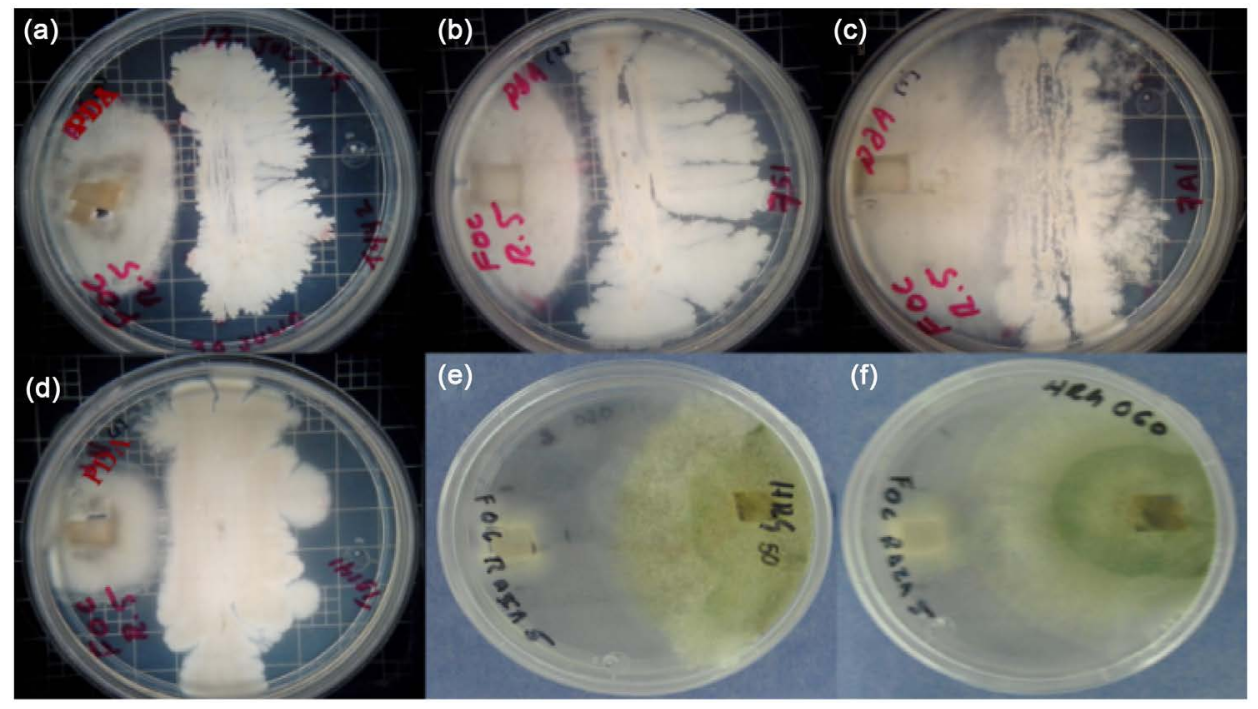

Figure 1. Antagonismo por pruebas de confrontación in vitro contra Fusarium oxysporum f. sp. ciceris raza 5. Inhibición por antibiosis de las bacterias antagonistas ((a)-(d)), a los 10 días de post-inoculación: (a) T442, Bacillus subtilis, b) 751, Agrobacterium radiobacter, (c) 7A1, Pseudomonas sp.; (d) T3141, Bacillus megaterium. Inhibición por competencia y micoparasitismo por las cepas fúngicas antagonistas ((e)-(f)), a los tres días de post-inoculación: (e) HRG-050, Trichoderma sp.; (f) HRG-060, Trichoderma sp. 
Bacillus. Los resultados de esta investigación también coinciden con los de Maciel et al. [45], ya que reportaron que cepas nativas de Trichoderma spp. y B. subtilis fueron antagonistas contra $F$. sambucinum, señalando que esta acción fue más pronunciada cuando se utilizó Trichoderma spp., el cual inhibió en más de $60 \%$ a las colonias del fitopatógeno. Por su parte, López et al. [40] reportan que cepas de Trichoderma y Bacillus nativas del noroeste de México tuvieron efectividad antagónica in vitro para control de la pudrición texana.

El biocontrol con cepas de Trichoderma sp. quizás se deba a la producción de enzimas, como viridin, trichodermin, tricodermina, tricotoxina, celobiasas, quitinazas y el compuesto 6-pentyl-apirona, que les otorga actividad antifúngica al penetrar y causar lisis del micelio de F. oxysporum, Martínez et al. [20]; sin embargo, diversos investigadores señalan que la competencia por espacio y nutrientes es una de las principales formas de ejercer el biocontrol sobre los patógenos del suelo, Infante et al. [17], Ezziyyani et al. [30] y Astorga et al. [46], tal como se observa en la Figure 1. Por otra parte, el antagonismo ejercido por ciertas cepas de Bacillus es por medio de antibiosis, competencia por espacio y nutrientes e inducción de resistencia sistémica; además, producen algunas enzimas que degradan la pared celular y que están involucradas en la actividad antagonista contra fitopatógenos, así como la producción de compuestos orgánicos volátiles, fitoalexinas y sideróforos, Bhattacharyya and Jha [22] y Singh and Singh [23].

\subsection{Efecto Protector de Las Cepas Antagonistas}

Con excepción de HRG-050, por inoculación de semillas con cinco antagonistas se logró incrementar de manera significativa el vigor de planta con respecto al testigo (Table 6); los mayores efectos se observaron con HRG-060 (Trichoderma sp.), T442 (B. subtilis) y T3141 (B. megaterium), los cuales superaron al testigo en $76 \%, 61 \%$ y $50 \%$, respectivamente, incluso en las plantas cultivadas con HRG-060 el vigor fue significa-

Table 6. Protección de plantas de garbanzo por inoculación de semillas con cepas antagonistas contra la fusariosis vascular en condiciones de campo.

\begin{tabular}{cccc}
\hline Cepas & Vigor de planta (\%) & Marchitez de follaje (\%) & Cáncer en raíz (\%) \\
\hline Testigo & $45.13 \mathrm{~d}^{*}$ & $64.20 \mathrm{ab}$ & $81.80 \mathrm{a}$ \\
Benomilo & $62.73 \mathrm{bc}$ & $64.13 \mathrm{ab}$ & $74.73 \mathrm{ab}$ \\
$7 \mathrm{~A} 1$ & $66.40 \mathrm{~b}$ & $51.40 \mathrm{~cd}$ & $65.67 \mathrm{bc}$ \\
T442 & $72.73 \mathrm{ab}$ & $48.87 \mathrm{~d}$ & $62.40 \mathrm{bc}$ \\
751 & $51.40 \mathrm{~cd}$ & $65.80 \mathrm{ab}$ & $83.00 \mathrm{a}$ \\
T3141 & $67.80 \mathrm{ab}$ & $61.60 \mathrm{bc}$ & $77.13 \mathrm{a}$ \\
HRG-050 & $44.00 \mathrm{~d}$ & $75.27 \mathrm{a}$ & $82.80 \mathrm{a}$ \\
HRG-060 & $79.53 \mathrm{a}$ & $45.80 \mathrm{~d}$ & $58.13 \mathrm{c}$ \\
C.V. & 11.53 & 10.549 & 10.889 \\
\hline
\end{tabular}

${ }^{\star}$ Letras no comunes indican diferencias estadísticamente significativas según Tukey $(P<0.05)$. 
tivamente mayor que el expresado por aquéllas tratadas con el producto Benomilo (testigo químico). En marchitez de follaje se observaron disminuciones significativas (28.7\% y $23.9 \%$ ) donde se aplicaron las cepas de HRG-060 y T442, respectivamente, en comparación al testigo. Mediante la inoculación de semillas con HRG-060, T442 y 7A1 el cáncer en raíz disminuyó en los respectivos 28.9\%, 23.7\% y $19.7 \%$ con respecto al testigo. Además, con HRG-060 (Trichoderma sp.) la disminución del cáncer fue 22.2\% mayor que lo que se obtuvo con Benomilo.

Estos resultados coinciden con diversos trabajos realizados a nivel de campo, donde se aplicó a estos organismos como antagonistas, reportándose una reducción significativa del cáncer en raíz, Karimi et al. [10], Moradi et al. [11], Manjunatha et al. [47] y Verma et al. [48]. De la misma manera, estos antagonistas han sido empleados en otros cultivos para protegerlos del ataque de fitopatógenos; al respecto, Arcos y Zúñiga [49] inocularon tubérculos de papa con cepas nativas de Bacillus, y lograron 60\% menos de bulbos infectados con Rhizoctonia y Spongospora.

El efecto protector de las cepas nativas de HRG-060 (Trichoderma sp.) y T442 (B. subtilis), quizás se deba a la producción de metabolitos y enzimas que ha sido documentada por diversos investigadores, como Qualhato et al. [50] que refieren que la mayoría de las especies de Trichoderma producen y secretan metabolitos tóxicos volátiles como $\beta$-1,3-glucanasa, quitinasa, fosfatasa ácida, celulasa y proteasas ácidas, que tienen efectos significativos sobre el crecimiento y desarrollo de los fitopatógenos, pues degradan su pared celular; asimismo, Tchameni et al. [51] que reportan que este género produce sideróforos que detienen el crecimiento de hongos patógenos; además se señala que la competencia por espacio y nutrientes, así como el micoparasitismo son sus principales mecanismos antagónicos, Ezziyyani et al. [30]. Otros investigadores afirman que el control biológico por Bacillus, es quizá por la producción de enzimas, metabolitos inhibitorios, antibióticos e inducción de la resistencia de la planta a los patógenos, Lima et al. [52] y Lee et al. [53]. Por su parte, Orberá et al. [54] reportan que esta bacteria es potencialmente antagónica a Fusarium sp. en cultivos ornamentales debido a la excreción de iturina, que actúa como antibiótico para fitopatógenos. Astorga et al. [46] demostraron que los procesos de competencia, antibiosis y parasitismo ejercidos por Trichoderma y Bacillus, lograron la inhibición y destrucción de Sclerotium cepivorum, Penicillium sp. y Pseudomonas marginalis.

\subsection{Efecto Estimulatorio del Crecimiento Vegetal Por Las Cepas Antagonistas}

La germinación de semillas inoculadas con las cepas antagonistas (Table 7) tuvo su mayor expresión (125\%, 125\% y 100\%) con las cepas T442 (B. subtilis), 751 (A. Radiobacter) y HRG-060 ( Trichoderma sp.) en comparación al testigo.

Estos resultados coinciden con lo reportado por Izzeddin y Medina [55], quienes al inocular semillas de diferentes hortalizas con cepas de Trichoderma, Pseudomonas y Bacillus, observaron mayor germinación de las semillas tratadas con respecto al testigo; asimismo, con los de León et al. [56], Chavarría y Carmona [57], quienes reportan incrementos significativos en la tasa de emergencia de soya y melina al emplear estos 
Table 7. Efecto en la germinación por la inoculación a las semillas de garbanzo con las cepas antagonistas.

\begin{tabular}{cc}
\hline Cepa & Germinación de semillas (\%) \\
\hline Testigo & $40.0 \mathrm{~d}$ \\
T442 & $90.0 \mathrm{a}^{*}$ \\
T3141 & $65.0 \mathrm{c}$ \\
7 A1 & $76.3 \mathrm{~b}$ \\
751 & $90.0 \mathrm{a}$ \\
HRG-050 & $40.0 \mathrm{~d}$ \\
HRG-060 & $80.0 \mathrm{ab}$ \\
C.V. & 7.0 \\
\hline
\end{tabular}

${ }^{\star}$ Letras diferentes en la columna indican diferencias estadísticas significativas, Tukey $(P<0.05)$.

microorganismos como inoculo en las semillas. Por su parte, Shahid et al. [58], aplicó Trichoderma en semillas de garbanzo y observaron germinación en más del $90 \%$ en laboratorio, superior en $15 \%$ al testigo. La estimulación en la germinación de semillas por estas cepas antagonistas puede atribuirse a la capacidad que tienen estos microorganismos de producir sustancias fisiológica y bioquímicamente activas, como giberelinas, citoquininas y ácido indolacético, las cuales estimulan la germinación de las semillas, Camelo et al. [59] y Sánchez et al. [60].

En cuanto a la altura de las plantas (Table 8), las cultivadas con 7A1 fueron las de menor porte con $27.5 \%$ menos en comparación al testigo, mientras que las demás fueron estadísticamente iguales a éste. La misma tendencia se observó en el diámetro de tallo, aunque con HRG-060 se tuvo un incremento de 14.9 y con T442 de $8.5 \%$. El verdor fue mayor con las cepas HRG-060, T442 y 7A1, con los respectivos incrementos de $12.8 \%, 11.7 \%, 12.6 \%$, comparados con el testigo. El peso fresco y seco tuvieron incrementos de $56.8 \%$ y $29.0 \%$ con la cepa HRG-060, mientras que el rendimiento por planta se incrementó $155 \%$ y $72 \%$ con las respectivas cepas de HRG-060 y T442. Con HRG060 también se tuvieron incrementos de $74.3 \%, 42.8 \%$ y $481 \%$ más en peso fresco, peso seco y rendimiento de grano por planta, en comparación a los promedios obtenidos con el tratamiento químico (Benomilo).

Los resultados anteriores coinciden con los de Shahid et al. [58] y Yadav et al. [61], ya que al inocular semillas de garbanzo con cepas de Trichoderma, reportaron incrementos significativos en altura y en peso seco de planta; asimismo, con los de Ávila et al. [9] y Verma et al. [48], quienes reportaron incrementos del rendimiento de grano del garbanzo después de inocular semillas con el mismo hongo. Por su parte, Verma y Yavad [62] inocularon semillas de garbanzo con cepas de Bacillus y reportaron incrementos significativos en el peso seco de raíz, parte aérea de la planta, y número de granos; mientras que Arcos y Zúñiga [49] inocularon papas con cepas nativas de Bacillus subtilis y observaron incrementos en altura de plantas, materia seca y rendimiento; De igual manera Jimtha et al. [63] notaron que Bacillus promueve notablemente el crecimiento en plantas de jengibre. 
Table 8. Comportamiento del crecimiento y rendimiento de plantas de garbanzo, cultivado en campo, por la inoculación de semillas con las cepas antagonistas.

\begin{tabular}{ccccccc}
\hline Tratamientos & $\begin{array}{c}\text { Altura de } \\
\text { planta }(\mathrm{cm})\end{array}$ & $\begin{array}{c}\text { Diámetro de } \\
\text { tallo }(\mathrm{mm})\end{array}$ & $\begin{array}{c}\text { Verdor } \\
(\text { SPAD })\end{array}$ & $\begin{array}{c}\text { Peso fresco } \\
(\mathrm{g})\end{array}$ & $\begin{array}{c}\text { Peso seco } \\
(\mathrm{g})\end{array}$ & $\begin{array}{c}\text { Rendimiento } \\
\left(\mathrm{g} \mathrm{planta}^{-1}\right)\end{array}$ \\
\hline Testigo & $28.0 \mathrm{a}^{*}$ & $4.7 \mathrm{ab}$ & $45.3 \mathrm{bc}$ & $19.9 \mathrm{bc}$ & $15.5 \mathrm{bc}$ & $10.0 \mathrm{~cd}$ \\
Benomilo & $23.5 \mathrm{ab}$ & $4.3 \mathrm{ab}$ & $42.3 \mathrm{c}$ & $17.9 \mathrm{c}$ & $14.0 \mathrm{c}$ & $4.4 \mathrm{e}$ \\
$7 \mathrm{~A} 1$ & $20.3 \mathrm{~b}$ & $4.3 \mathrm{~b}$ & $51.0 \mathrm{a}$ & $19.6 \mathrm{bc}$ & $13.9 \mathrm{c}$ & $6.5 \mathrm{de}$ \\
T442 & $28.2 \mathrm{a}$ & $5.1 \mathrm{ab}$ & $50.6 \mathrm{a}$ & $23.6 \mathrm{~b}$ & $17.5 \mathrm{ab}$ & $17.2 \mathrm{~b}$ \\
751 & $26.3 \mathrm{ab}$ & $4.9 \mathrm{ab}$ & $40.6 \mathrm{c}$ & $20.8 \mathrm{bc}$ & $15.3 \mathrm{bc}$ & $13.0 \mathrm{bc}$ \\
T3141 & $23.5 \mathrm{ab}$ & $5.0 \mathrm{ab}$ & $43.7 \mathrm{c}$ & $21.2 \mathrm{bc}$ & $15.5 \mathrm{bc}$ & $12.7 \mathrm{bc}$ \\
HRG-050 & $25.0 \mathrm{ab}$ & $4.9 \mathrm{ab}$ & $40.9 \mathrm{c}$ & $17.2 \mathrm{c}$ & $13.6 \mathrm{c}$ & $4.8 \mathrm{e}$ \\
HRG-060 & $28.1 \mathrm{a}$ & $5.4 \mathrm{a}$ & $51.1 \mathrm{a}$ & $31.2 \mathrm{a}$ & $20.0 \mathrm{a}$ & $25.6 \mathrm{a}$ \\
C.V. & 14.3 & 11.0 & 6.4 & 10.8 & 9.6 & 23.2 \\
\hline
\end{tabular}

${ }^{\star}$ Letras diferentes en la columna indican diferencias estadísticas significativas, Tukey $(P<0.05)$.

El efecto estimulatorio en el crecimiento y rendimiento del garbanzo por parte de Trichoderma (HRG-060), quizás se deba a las hormonas que sintetiza (ácido indol-3acético, auxinas, giberelinas y citoquininas), a la producción de sideróforos, solubilización de fosfatos y magnesio, eficiencia en el uso del nitrógeno, producción de ácido glucónico y ácido cítrico, aumento de la solubilización de micronutrientes (hierro y manganeso) o a la actividad antagonista contra $F$. oxysporum, tal como lo señalan numerosos investigadores [19] [48] [61] [64]. Por otra parte, la capacidad de estimulación del crecimiento por B. subtilis (T442), también puede ser consecuencia de las vitaminas que sintetiza, de la fijación del nitrógeno atmosférico y la solubilización de fosfatos que las plantas aprovechan [49] [59] [60].

\section{Conclusiones}

Las cepas T442, 751 y 7A1 correspondieron a Bacillus subtilis, Agrobacterium radiobacter y Pseudomonas sp., con un porcentaje de confiabilidad superior al 90\%, HRG050 y HRG-060 fueron identificadas como Trichoderma sp, mientras que T3141 correspondió a Bacillus megaterium, con bajo porcentaje de confiabilidad.

En las pruebas in vitro, la mayor inhibición del crecimiento micelial de Foc raza 5 fue con HRG-060, HRG-050, T442 y 751, con un PICR mayor al 50\%, demostrando así su efecto antagonista, por lo que el uso de estos microorganismos en garbanzo, como biofumigantes y biofertilizantes, es promisorio bajo el contexto ambiental del centro de Sinaloa.

La inoculación de semillas con todos los aislamientos, excepto HRG-050, favoreció la germinación, y los mejores resultados se lograron al emplear como inoculo a T442, 751 y HRG-060, pero en campo las mejores respuestas se obtuvieron con HRG-060 y T442, ya que con éllos se incrementó el vigor de planta (grosor del tallo, verdor y biomasa), disminuyó la fusariosis y, en consecuencia, se incrementó el rendimiento de grano por planta. 


\section{References}

[1] Jukanti, A.K., Gaur, P.M., Gowda, C.L. and Chibbar, R.N. (2012) Nutritional Quality and Health Benefits of Chickpea (Cicer arietinum L.): A Review. British Journal of Nutrition, 108, S11-S26. https://doi.org/10.1017/s0007114512000797

[2] Jiménez-Díaz, R.M., Castillo, P., Jiménez-Gasco, M.M., Landa, B.B. and Navas-Cortes, J.A. (2015) Fusarium Wilt of Chickpeas: Biology, Ecology and Management. Crop Protection, 73, 16-27. https://doi.org/10.1016/j.cropro.2015.02.023

[3] FAOSTAT-Food and Agriculture Organization of the United Nations (2015) Consultado en Octubre de 2015. http://www.fao.org

[4] Guerrero, A.B.Z., Acosta, G.J.A., Sánchez, G.B.M., Ortega, M.P.F. and González, C.M.M. (2015) Razas patogénicas de Fusarium oxysporum f. sp. ciceris en garbanzo cultivado en Guanajuato, México. Revista Fitotecnia Mexicana, 38, 183-190.

[5] SIAP, SAGARPA (2015) Servicio de Información Agropecuaria y Pesquera. Consultado en Mayo de 2016. http://www.siap.gob.mx/avance-de-siembras-y-cosechas

[6] Navas-Cortés, J.A., Hau, B. and Jiménez, D.R.M. (2000) Yield Loss in Chickpeas in Relation to Development of Fusarium Wilt Epidemics. Phytopathology, 90, 1269-1278. https://doi.org/10.1094/PHYTO.2000.90.11.1269

[7] Demers, J.E., Garzon, C.D. and Jimenez-Gasco, M.M. (2014) Striking Genetic Similarity between Races of Fusarium oxysporum f. sp. ciceris Confirms a Monophyletic Origin and Clonal Evolution of the Chickpea Vascular Wilt Pathogen. European Journal of Plant Pathology, 139, 303-318. https://doi.org/10.1007/s10658-014-0387-8

[8] Velarde, F.S., Ortega, M.P.F., Fierros, L.G.A., Padilla, V.I., Gutierres, P.E., Rodríguez, C.F.G., López, V.J.A., Acosta, G.J.A. and Garzón, T.J.A. (2015) Identificación molecular y biológica de las razas 0 y 5 de Fusarium oxysporum Schlechtend.: Fr f. sp. ciceris (Padwick) Matuo \& K. Sato del garbanzo en el noroeste de México. Revista Mexicana de Ciencias Agrícolas, 6, 735-748.

[9] Ávila, M.J.A., Padilla, Z.G., Martínez, H.D., Rivas, S.F.J., Coronado, E.M.A. and Ortega, M.P. (2015) Respuesta de algunos componentes del rendimiento del cultivo de garbanzo (Cicer arietinum L.) a la inoculación de Mesorhizobium ciceri, Trichoderma harzianum y Bacillus subtilis en la región agrícola de la costa de Hermosillo. Biotecnia, 17, 3-8. https://doi.org/10.18633/bt.v17i3.212

[10] Karimi, K., Amini, J., Harighi, B. and Bahramnejad, B. (2012) Evaluation of Biocontrol Potential of Pseudomonas and Bacillus spp. against Fusarium Wilt of Chickpea. Australian Journal of Crop Science, 6, 695-703.

[11] Moradi, H., Bahramnejad, B., Amini, J., Siosemardeh, A. and Haji-Allahverdipoor, K. (2012) Suppression of Chickpea (Cicer arietinum L.) Fusarium Wilt by Bacillus subtilis and Trichoderma harzianum. Plant Omics Journal, 5, 68-74.

[12] Naher, L., Yusuf, U.K., Ismail, A. and Hossain, K. (2014) Trichoderma spp.: A Biocontrol Agent for Sustainable Management of Plant Diseases. Pakistan Journal of Botany, 46, 14891493.

[13] Pastrana, A.M., Basallote-Ureba, M.J., Aguado, A., Akdi, K. and Capote, N. (2016) Biological Control of Strawberry Soil-Borne Pathogens Macrophomina phaseolina and Fusarium solani, Using Trichoderma asperellum and Bacillus spp. Phytopathologia Mediterranea, 55, 109-120.

[14] Abdulkareem, M., Aboud, M.H., Saood, M.H. and Shibly, K.M. (2014) Antagonistic Activity of Some Plant Growth Rhizobacteria to Fusarium graminearum. International Journal of Phytopathology, 3, 49-54. 
[15] Hernández, R.A., Ruíz, B.Y., Acebo, G.Y., Miguélez, S.Y. and Heydrich, P.M. (2014) Antagonistas microbianos para el manejo de la pudrición negra del fruto en Theobroma cacao L. Estado actual y perspectivas de uso en Cuba. Protección Vegetal, 29, 11-19.

[16] Eshetu, B., Amare, A. and Seid, A. (2015) Antagonistic Effect of Native Bacillus Isolates against Black Root Rot of Faba Bean. African Crop Science Journal, 23, 249-259.

[17] Infante, D., Martínez, B., González, N. and Reyes, Y. (2009) Mecanismos de acción de Trichoderma frente a hongos fitopatógenos. Revista de Protección Vegetal, 24, 14-21.

[18] Melnick, R.L., Suárez, C., Bailey, B.A. and Backman, P.A. (2011) Isolation of Endophytic Endospore-Forming Bacteria from Theobroma cacao as Potential Biological Control Agents of Cacao Diseases. Biological Control, 57, 236-245. https://doi.org/10.1016/j.biocontrol.2011.03.005

[19] Vinale, F., Sivasithamparam, K., Ghisalberti, E.L., Marra, R., Barbetti, M.J., Li, H., Woo, S.L. and Lorito, M. (2008) A Novel Role of Trichoderma Secondary Metabolites in the Interactions with Plants. Physiological and Molecular Plant Pathology, 72, 80-86. https://doi.org/10.1016/j.pmpp.2008.05.005

[20] Martínez, B., Infante, D. and Reyes, Y. (2013) Trichoderma spp. y su función en el control de plagas en los cultivos. Revista de Protección Vegetal, 28, 1-11.

[21] Ruiz, S.E., Cristóbal, A.J., Reyes, R.A., Tun, S.J., García, R.A. and Pacheco, A.J. (2014) In Vitro Antagonistic Activity of Bacillus subtilis Strains Isolated from Soils of the Yucatan Peninsula against Macrophomina phaseolina and Meloidogyne incognita. International Journal of Experimental Botany, 83, 45-47.

[22] Bhattacharyya, P.N. and Jha, D.K. (2012) Plant Growth-Promoting Rhizobacteria (PGPR): Emergence in Agriculture. World Journal of Microbiology and Biotechnology, 28, 1327 1350. https://doi.org/10.1007/s11274-011-0979-9

[23] Singh, J.S. and Singh, D.P. (2013) Plant Growth Promoting Rhizobacteria (PGPR): Microbes in Sustainable Agriculture. In: Malik, A., Grohmann, E. and Alves, M., Eds., Management of Microbial Resources in the Environment, Springer, Berlin, 361-385. https://doi.org/10.1007/978-94-007-5931-2_14

[24] Cano, M.A. (2011) Interacción de microorganismos benéficos en plantas: Micorrizas, Trichoderma spp. y Pseudomonas spp. Una Revisión. Revista UDCA Actualidad \& Divulgación Científica, 14, 15-31.

[25] Calvo, A.J.A., Rivera, C.G., Orozco, C.S. and Orozco, R.R. (2012) Aislamiento Ezziyyaniy evaluación in Vitro de antagonistas de Botrytis cinerea en mora. Agronomía Mesoamericana, 23, 225-231. https://doi.org/10.15517/am.v23i2.6481

[26] Robinson-Boyera, L., Jegerb, J., Xu, M. and Jeffries, P. (2009) Management of Strawberry Grey Mould Using Mixtures of Biocontrol Agents with Different Mechanisms of Action. Biocontrol Science and Technology, 19, 1051-1065. https://doi.org/10.1080/09583150903289105

[27] Agurto, S.T. (1989) Manual de Técnicas en Microbiología. Talleres Gráficos de Imprenta L Pluma Fuente. Lima, 256 p.

[28] Garassini, L.A. (1967) Microbiología Agrícola. Imprenta Universitaria, Maracaibo, 646 p.

[29] Kirk, P.M. and Ainsworth, G.C. (2008) Ainsworth \& Bisby's Dictionary of the Fungi. 10th Edition, CSIRO Publishing, Collingwood, $771 \mathrm{p}$.

[30] Ezziyyani, M., Pérez, S.C., Sid, A.A., Requena, M.E. and Candela, M.E. (2004) Trichoderma harzianum como fungicidas para el control de Phytophtora capsici en plantas de pimiento (Capsicum annuum L.). Anales de Biología, 26, 35-45.

[31] Estrella, F.S., Elorrieta, M.A., Vargas, G.C., Lopez, M.J. and Moreno, J. (2001) Selective Iso- 
lation of Antagonist Microorganisms of Fusarium oxysporum f. sp. melonis. Biological Control of Fungal and Bacterial Plant Pathogens, International Organization for Biological Control (IOBC) West Palaeartic Regional Sector (WPRS), Bulletin, 24, 109-112.

[32] Ko, H.S., Jin, R.D., Krishnan, H.B., Lee, S.B. and Kim, K.Y. (2009) Biocontrol Ability of $L y$ sobacter Antibioticus HS124 against Phytophthora blight Is Mediated by the Production of 4-Hydroxyphenylacetic acid and Several Lytic Enzymes. Current Microbiology, 59, 608615. https://doi.org/10.1007/s00284-009-9481-0

[33] Ortigoza, F.J. and Ruiloba, L.S.L. (1998) Microbiología Práctica. Departamento de Microbiología. 2nd Edition, ENCB-IPN, México, 205 p.

[34] Ferron, P. (1981) Pest Control by the Fungi Beauveria and Metarhizium. In: Burge, H.D., Ed., Microbial Control of Pests and Plant Diseases 1970-1980, Academic Press, New York, 465-481.

[35] Palleroni, N.J. (2005) Bergey's Manual of Systematic Bacteriology, Vol. 2, Part B. Springer, New York, 323-370.

[36] Larrea, I.I., Falconí, B.C. and Arcos, A.A. (2015) Aislamiento y caracterización de cepas de Bacillus spp. con actividad contra Tetranychus urticae Koch en cultivos comerciales de rosas. Revista Colombiana de Biotecnología, 17, 140-148.

https://doi.org/10.15446/rev.colomb.biote.v17n2.54291

[37] Sosa, L.A.I., Álvarez, R.V.P., Torres, C.D. and Casadesús, R.L. (2011) Identificación y caracterización de seis aislados pertenecientes al género Bacillus promisorios para el control de Rhizoctonia Solani Künh y Sclerotium Rolfsii Sacc. Fitosanidad, 15, 39-43.

[38] Álvarez, L.C., Osorio, V.W., Díez, G.M.C. and Marín, M.M. (2014) Caracterización bioquímica de microorganismos rizosféricos de plantas de vainilla con potencial como biofertilizantes. Agronomía Mesoamericana, 25, 225-241.

https://doi.org/10.15517/am.v25i2.15426

[39] Gacitúa, A., Valiente, S.F., Díaz, C.P., Hernández, K.C., Uribe, J.M.M. and Sanfuentes, V.E. (2009) Identification and Biological Characterization of Isolates with Activity Inhibitive against Macrophomina phaseolina (Tassi) Goid. Chilean Journal of Agricultural Research, 69, 526-533. https://doi.org/10.4067/S0718-58392009000400007

[40] López, V.B.E., Armenta, B.A.D., Hernández, V.S., Apodaca, S.M.A., Samaniego, G.J.A., Leyva, M.K.Y. and Valdez, O.A. (2015) Selección in Vitro e identificación de aislados de Trichoderma spp. y Bacillus spp. nativos para el control de Phymatotrichopsis omnívora. Información Técnica Económica Agraria, 111, 310-325.

[41] Aponte, G.Y., Salazar, L.A., Alcano, M.J., Sanabria, N.H., Guzmán, J.J. and Gámez, A.J. (2012) Evaluación en condiciones in Vitro de la masa micelial de hongos fitopatógenos mediante el uso de filtrados de aislamientos de Trichoderma spp. Agronomía Trop, 62, $17-24$.

[42] Ríos, V.C., Caro, C.J.M., Berlanga, R.D.I., Ruiz, C.M.F., Ornelas, P.J.J., Salas, M.M.A., Villalobos, P.E. and Guerrero, P.V.M. (2016) Identification and Antagonistic Activity in Vitro of Bacillus spp. and Trichoderma spp. Isolates against Common Phytopathogenic Fungi. Revista Mexicana de Fitopatología, 34, 84-99.

[43] Gajbhiye, A., Rai A.R., Meshram, S.U. and Dongre, A.B. (2010) Isolation, Evaluation and Characterization of Bacillus subtillis from Cotton Rhizospheric Soil with Biocontrol Activity against Fusarium oxysporum. World Journal of Microbiology and Biotechnology, 26, 1187-1194. https://doi.org/10.1007/s11274-009-0287-9

[44] Kumar, P.D., Thenmozhi, R., Anupama, P.D., Nagasathya, A., Thajuddin, N. and Paneerselvam, A. (2012) Selection of Potential Antagonistic Bacillus and Trichoderma Isolates from Tomato Rhizospheric Soil against Fusarium oxysporum f. sp. lycoperscisi. Research 
Journal of Biological Sciences, 6, 523-531. https://doi.org/10.3923/rjbsci.2011.523.531

[45] Maciel, C.G., Walke, C., Muniz, M.F.B. and Araujo, M.M. (2014) Antagonismo de Trichoderma spp. e Bacillus subtilis (UFV3918) a Fusarium sambucinum em Pinus elliottii engelm. Revista Árvore, 38, 505-512. https://doi.org/10.1590/S0100-67622014000300013

[46] Astorga, Q.K., Meneses, M.K., Zúñiga, V.C., Brenes, M.J. and Rivera, M.W. (2014) Evaluación del antagonismo de Trichoderma sp. y Bacillus subtilis contra tres patógenos del ajo. Revista Tecnología en Marcha, 27, 82-91. https://doi.org/10.18845/tm.v27i2.1929

[47] Manjunatha, S.V., Naik, M.K., Khan, M.F.R. and Goswami, R.S. (2013) Evaluation of Bio-Control Agents for Management of Dry Root Rot of Chickpea Caused by Macrophomina phaseolina. Crop Protection, 45, 147-150. https://doi.org/10.1016/j.cropro.2012.09.003

[48] Verma, J.P., Yadav, J., Tiwari, K.N. and Jaiswal, D.K. (2014) Evaluation of Plant Growth Promoting Activities of Microbial Strains and Their Effect on Growth and Yield of Chickpea (Cicer arietinum L.) in India. Soil Biology and Biochem, 70, 33-37. https://doi.org/10.1016/j.soilbio.2013.12.001

[49] Arcos, J. and Zúñiga, D. (2016) Rizobacterias promotoras de crecimiento de plantas con capacidad para mejorar la productividad en papa. Revista Latinoamericana de la Papa, 20, 18-31.

[50] Qualhato, T.F., Lopes, F.A., Steindorff, A.S., Brandao, R.S., Jesuino, R.S. and Ulhoa, C.J. (2013) Mycoparasitism Studies of Trichoderma Species against Three Phytopathogenic Fungi: Evaluation of Antagonism and Hydrolytic Enzyme Production. Biotechnology Letters, 35, 1461-1468. https://doi.org/10.1007/s10529-013-1225-3

[51] Tchameni, S.N., Ngonkeu, M.E., Begoude, B.A.D., Wakam, N.L., Owona A.D., Mbarga, J.B., Tchana, T., Tondje, P.R., Etoa, F.X. and Kuaté, J. (2011) Effect of Trichoderma asperellum and Arbuscular Mycorrhizal Fungi on Cacao Growth and Resistance against Black Pod Disease. Crop Protection, 30, 1321-1327. https://doi.org/10.1016/j.cropro.2011.05.003

[52] Lima, O.D.R., Oliveira, L.J.M.G., Silva, M.S.B.S. and Rodrigues, A.A.C. (2014) Ação antifúngica in Vitro de isolados de Bacillus spp sobre Fusarium oxysporum f. sp. lycopersici. Revista Caatinga, 27, 57-64.

[53] Lee, Y.S. and Kim, K.Y. (2016) Antagonistic Potential of Bacillus pumilus L1 against Root-Knot Nematode, Meloidogyne arenaria. Journal of Phytopathology, 164, 29-39. https://doi.org/10.1111/jph.12421

[54] Orberá, R.T.M., Serrat, D.M.J. and González, G.Z. (2009) Potencialidades de bacterias aerobias formadoras de endosporas para el biocontrol en plantas ornamentales. Fitosanidad, 13, 95-100.

[55] Izzeddin, N.A. and Medina, T.L. (2011) Efecto del control biológico por antagonistas sobre fitopatógenos en vegetales de consumo humano. Rev. de la Facultad de Ciencias de la Salud, Universidad de Carabobo, 15, 8-12.

[56] León, M., Yaryura, P.M., Montecchia, M.S., Hernández, A.I., Correa, O.S., Pucheu, N.L., Kerber, N.L. and García, A.F. (2009) Antifungal Activity of Selected Indigenous Pseudomonas and Bacillus from the Soybean Rhizosphere. International Journal of Microbiology, 2009, Article ID: 572049. https://doi.org/10.1155/2009/572049

[57] Chavarría, V.M. and Carmona, S.R. (2016) Efecto de microorganismos antagonistas en el control de la enfermedad denominada "Nectria" en la melina Gmelina arborea Roxb. Revista Forestal Mesoamericana Kurú, 21-29. https://doi.org/10.18845/rfmk.v0i0.2549

[58] Shahid, M., Singh, A., Srivastava, M., Sachan, C.P. and Biswas, S.K. (2011) Effect of Seed Treatment on Germination and Vigour in Chickpea. Trends in Biosciences, 4, 205-207. 
[59] Camelo, M., Vera, S.P. and Bonilla, R. (2011) Mecanismos de acción de las rizobacterias promotoras del crecimiento vegetal. Rev. Corpoica-Ciencia y Tecnol. Agropecuaria, 12, 159-166.

[60] Sánchez, L.D.B., García H.A.M., Romero P.F.A. and Bonilla B.R.R. (2014) Efecto de rizobacterias promotoras de crecimiento vegetal solubilizadoras de fosfato en Lactuca sativa cultivar White Boston. Revista Colombiana de Biotecnología, 16, 122-128.

https://doi.org/10.15446/rev.colomb.biote.v16n2.41077

[61] Yadav, J., Verma, J.P. and Tiwari, K.N. (2011) Plant Growth Promoting Activities of Fungi and Their Effect on Chickpea Plant Growth. Asian Journal of Biological Sciences, 4, 291 299. https://doi.org/10.3923/ajbs.2011.291.299

[62] Verma, J.P. and Yadav, J. (2012) Evaluation of Plant Growth Promoting Rhizobacteria and Their Effect on Plant Growth and Grain Yield of Chickpea (Cicer arietinum L.) under Sustainable Agriculture Production. IJREISS, 2, 51-57.

[63] Jimtha, J.C., Jishma, P., Arathy, G.B., Anisha, C. and Radhakrishnan, E.K. (2016) Identification of Plant Growth Promoting Rhizosphere Bacillus sp. WG4 Antagonistic to Pythium myriotylum and Its Enhanced Antifungal Effect in Association with Trichoderma. Journal of Soil Science and Plant Nutrition, Epub.

[64] Harman, G.E. (2011) Multifunctional Fungal Plant Symbionts: New Tools to Enhance Plant Growth and Productivity. New Phytologist, 189, 647-652.

https://doi.org/10.1111/j.1469-8137.2010.03614.x

Submit or recommend next manuscript to OALib Journal and we will provide best service for you:

- Publication frequency: Monthly

- 9 subject areas of science, technology and medicine

- Fair and rigorous peer-review system

- Fast publication process

- Article promotion in various social networking sites (LinkedIn, Facebook, Twitter, etc.)

- Maximum dissemination of your research work

Submit Your Paper Online: Click Here to Submit

Or Contact service@oalib.com 